\title{
A traveling-wave THz photomixer based on angle-tuned phase matching
}

\author{
Shuji Matsuuraa) and Geoffrey A. Blake \\ Division of Geological and Planetary Sciences, California Institute of Technology, Pasadena, \\ California 91125 \\ Rolf A. Wyss and J. C. Pearson \\ Jet Propulsion Laboratory, California Institute of Technology, Pasadena, California 91109 \\ Christoph Kadow, Andrew W. Jackson, and Arthur C. Gossard \\ Materials Department, University of California, Santa Barbara, California 93106
}

(Received 5 January 1999; accepted for publication 12 March 1999)

A traveling-wave $\mathrm{THz}$ photomixer based on a free-space optical- $\mathrm{THz}$ phase-matching scheme is proposed. A dc-biased coplanar strip line fabricated on low-temperature-grown GaAs serves as the active area of the device, and is illuminated by two noncollinear laser beams which generate interference fringes that are accompanied by $\mathrm{THz}$ waves. The device with the laser-power-handling capability over $300 \mathrm{~mW}$ and a 3-dB bandwidth of $1.8 \mathrm{THz}$ was experimentally demonstrated. The results show that traveling-wave photomixers have the potential to surpass small-area designs. (C) 1999 American Institute of Physics. [S0003-6951(99)03719-5]

The difference frequency generation of $\mathrm{THz}$ waves by optical-heterodyne (photo) mixing in ultrafast photoconductors, in particular, those based on low-temperature-grown (LTG) GaAs, has been widely investigated as a highly tunable, compact, solid-state $\mathrm{THz}$ source for high-resolution molecular spectroscopy and as a local oscillator for heterodyne receivers in remote-sensing applications. ${ }^{1-5}$

For conventional photomixers, the output power is proportional to the square of the photocurrent, and the bandwidth is limited by the photocarrier lifetime and the electrode capacitance. ${ }^{1}$ Therefore, photomixers have been designed to have narrow electrode gaps for high photocurrent and small active areas $\left(\sim 10^{2} \mu \mathrm{m}^{2}\right)$ for small capacitance. However, such designs have limited power handling capabilities, ${ }^{5}$ thus restricting the $\mathrm{THz}$ output power. In order to overcome this difficulty, we propose a large-area traveling-wave photomixer design. The traveling-wave photomixer not only has high-power-handling capability, but its bandwidth is also not limited by the electrode capacitance because of the nature of the distributed circuit elements. As high-power laser sources are improved, the traveling-wave photomixer can provide good $\mathrm{THz}$ output with wide bandwidths, and should ultimately be superior to conventional small-area photomixers.

The present device consists of a dc-biased coplanar strip line terminated by an antenna fabricated on LTG GaAs. The $\sim 10^{3} \mu \mathrm{m}^{2}$ active area is illuminated by two laser beams tilted at an angle with respect to each other in order to generate optical interference fringes moving along the strip line. The heterodyne mixing process generates photocarrier density waves which are oscillating at the difference frequency, and which are accompanied by $\mathrm{THz}$ traveling waves. If the velocity of the optical fringes and the group velocity of the $\mathrm{THz}$ wave are equalized, the $\mathrm{THz}$ waves from the distributed area are coherently superposed and effectively emitted by the antenna. Several authors have achieved velocity (phase) matching between optical waves and microwaves in traveling-wave optical waveguide photodetectors, which slow down the microwave velocity with periodic capacitance loading of the microwave transmission line. ${ }^{6,7}$ Such a phasematched waveguide photomixer requires careful design and fabrication because of its fixed structure, and as a result, these devices have not yet been extended to the $\mathrm{THz}$ range.

The free-space traveling-wave photomixer shown in Fig. 1 does not require such complicated waveguide structures because the phase matching is fulfilled by tuning the incident angle of the two laser beams. When a plane wave of frequency $f_{1}$ arrives at the photomixer surface at an angle of incidence $\theta_{1}$, the optical wave propagation constant along the surface is given by $k_{1}=2 \pi f_{1} \sin \theta_{1} / c$, where $c$ is the velocity of light. If another wave with a different frequency $f_{2}$ and an angle of incidence $\theta_{2}$, which has a propagation constant of $k_{2}=2 \pi f_{2} \sin \theta_{2} / c$, is superposed on the same spot as the first wave, an interference fringe pattern oscillating at the difference frequency $f=f_{1}-f_{2}$ is formed. Since absorption occurs in a thin surface layer (penetration depth $\sim 1 \mu \mathrm{m}$ ), the photocarriers have the same spatial distribution

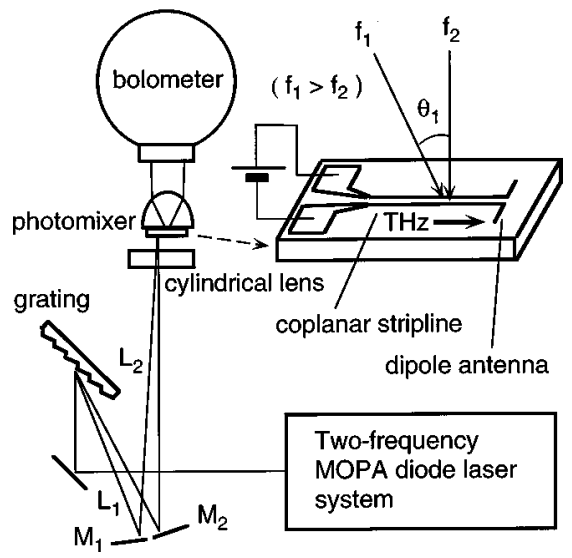

FIG. 1. Experimental setup and schematic device structure.

${ }^{a}$ Electronic mail: matsuura@gps.caltech.edu 
as the optical interference fringes. The velocity of the interference fringes along the surface is given by

$$
v_{\mathrm{op}}=\frac{2 \pi\left(f_{1}-f_{2}\right)}{k_{1}-k_{2}}=\frac{\left(f_{1}-f_{2}\right) c}{f_{1} \sin \theta_{1}-f_{2} \sin \theta_{2}} .
$$

When $f_{1}>f_{2}$ and $k_{1}>k_{2}$, the wave propagation direction is parallel to $k_{1}$. It is clear that the velocity can be tuned by changing the angle of incidence of the optical beams. On the other hand, the group velocity of a $\mathrm{THz}$ wave generated in a dispersionless transmission line is given by

$$
v_{\mathrm{THz}}=c / \sqrt{\left(1+\epsilon_{r}\right) / 2},
$$

where $\epsilon_{r}$ is the dielectric constant of the substrate material. When $v_{\mathrm{op}}=v_{\mathrm{THz}}$, the generated $\mathrm{THz}$ waves sum coherently.

In the setup shown in Fig. 1, the optical source is a two-frequency master oscillator power amplifier (MOPA) $850 \mathrm{~nm}$ semiconductor laser system. A tapered semiconductor optical amplifier is simultaneously injection seeded by a tunable external cavity laser $\left(\lambda_{1}=848-853 \mathrm{~nm}\right)$ and a cavity-locked fixed-frequency laser $\left(\lambda_{2}=854 \mathrm{~nm}\right)$, and provides a maximum two-frequency power of $500 \mathrm{~mW}{ }^{8}$ The output from the MOPA is split into two beams by a 2000 groove $/ \mathrm{mm}$ diffraction grating. The advantage of the split collinear beam over two individual lasers is the nearly identical spatial-mode quality of the two colors, which is essential for efficient $\mathrm{THz}$ wave generation.

For a grating incidence angle of $\alpha=50^{\circ}$, the diffraction angle and its dispersion is $\beta=70^{\circ}$ and $d \beta / d f$ $=0.8^{\circ} \mathrm{THz}^{-1}$, respectively. The diffracted beams are reflected by two mirrors and focused onto the photomixer by a 6-mm-focal-length cylindrical lens. The $\sim 2$-mm-diam circular MOPA beam is compressed to a $\sim 0.4 \mathrm{~mm} \times 2 \mathrm{~mm}$ elliptical beam by the grating. The maximum available laser power after the grating is $\sim 300 \mathrm{~mW}$.

In the present implementation, the incidence angle of the fixed laser is set to zero $\left(\theta_{2}=0\right)$. For GaAs $\left(\epsilon_{r}=12.8\right)$, the phase-matching condition is fulfilled when the angle separation between the two laser beams (hereafter, the beam tilt angle), $\theta_{1}$, is approximately half the dispersion angle of the two beams, or $\theta_{1} \cong 0.5 \Delta \beta$. Mirror $M_{1}$ is adjusted to overlap the two laser beams at the photomixer surface. The THz output power was measured with a silicon composite bolometer. Thermal emission caused by the laser and electrical power was estimated from the bolometer signal when one laser beam was blocked.

The photomixer used here is fabricated on a $1-\mu \mathrm{m}$-thick LTG-GaAs film grown on semi-insulating GaAs substrates by molecular beam epitaxy (MBE) at a temperature of $225^{\circ} \mathrm{C}$. The material was annealed ex situ by rapid thermal annealing (RTA) for $30 \mathrm{~s}$ at $600{ }^{\circ} \mathrm{C}$ in a forming gas environment. The photocarrier lifetime of the material measured by time-resolved reflectance was $0.28 \mathrm{ps}$. The Ti/Pt/Au metal electrodes $(0.02 / 0.02 / 0.2 \mu \mathrm{m}$ thickness) fabricated on the LTG GaAs consist of a $2-\mu \mathrm{m}$ gap and $4-\mu \mathrm{m}$-wide coplanar strip line. The strip line is terminated by a $47-\mu \mathrm{m}$-long and 5 - $\mu$ m-wide dipole antenna. A hyperhemispherical highresistivity silicon lens is attached to the backside of the dipole antenna to collimate the output.

Figure 2 presents the bias voltage dependence of the dc photocurrent of the photomixer and the $\mathrm{THz}$ output meaDownloaded 08 Mar 2006 to 131.215.225.174. Redistribution subje

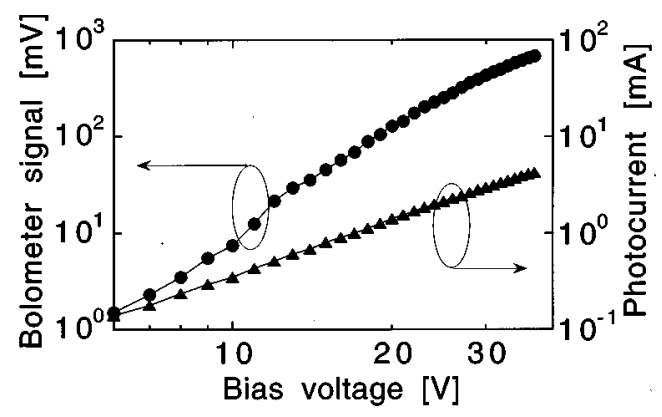

FIG. 2. The bias voltage dependence of the dc photocurrent (triangles) and the THz output at $f=1 \mathrm{THz}$ (circles).

sured at $1 \mathrm{THz}$ for the laser power of $300 \mathrm{~mW}$. The $\mathrm{dc}$ photocurrent shows quadratic dependence, whereas linear (Ohmic) dependence is expected. The quadratic dependence of the photocurrent has been seen in LTG-GaAs photomixers with sub- $\mu \mathrm{m}$ electrodes at high bias fields $\left(E>10^{5} \mathrm{~V} / \mathrm{cm}\right)$, where the space-charge-limited currents take place. ${ }^{2,5}$ The data show a quadratic dependence even at low biases $(E$ $\sim 2 \times 10^{4} \mathrm{~V} / \mathrm{cm}$ ), which might arise here, because the photon flux per unit area is much lower than that for small-area photomixers.

For a free-space traveling-wave photomixer, tuning of the beam tilt angle should strongly affect the $\mathrm{THz}$ output. Figure 3(a) presents the $\mathrm{THz}$ output dependence on the beam tilt angle, again measured at $1 \mathrm{THz}$. The data show a maximum at $\theta_{1}=0.42^{\circ}$, which is close to that expected. The angular width $\Delta \theta_{1}$ was almost independent of the THz-wave frequency. In Fig. 3(b), the phase-matching angle is plotted versus frequency. The data show good agreement with the predicted tuning coefficient of $0.42^{\circ} \mathrm{THz}^{-1}$, indicated by the solid line. These results demonstrate that the present device works as a (phase-matched) distributed device.

The THz output spectrum is shown in Fig. 4. As noted above, the bandwidth of the ideal traveling-wave photomixer should be determined mainly by the LTG-GaAs carrier lifetime, which rolls off as $6 \mathrm{~dB} /$ Oct. However, the data show a 3-dB bandwidth of $1.8 \mathrm{THz}$, which exceeds the carrierlifetime-limited bandwidth of $600 \mathrm{GHz}$, and a roll off of $\sim 12$ $\mathrm{dB} /$ Oct, similar to that of small-area photomixers. ${ }^{1}$ The additional roll off can be interpreted as that arising from the
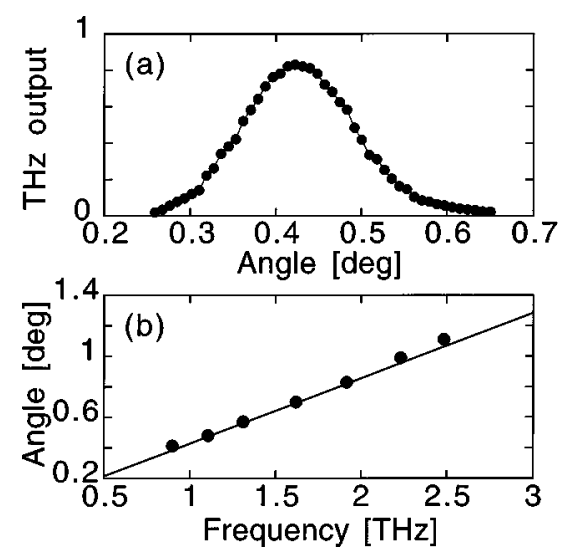

FIG. 3. (a) THz output dependence on the beam tilt angle measured at $f$ $=1 \mathrm{THz}$. (b) The phase-matching angle vs frequency. The solid line is that

calculated from Eqs. (1) and (2).
to AIP license or copyright, see http://apl.aip.org/apl/copyright.jsp 


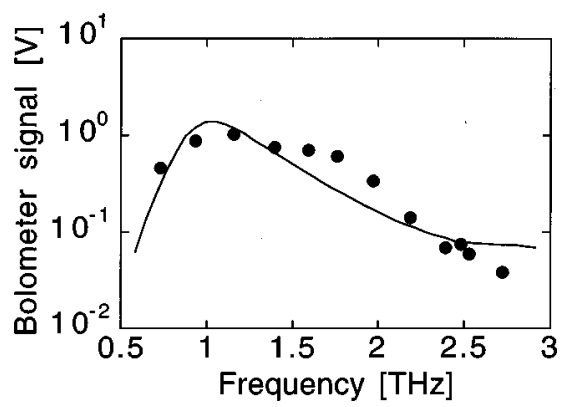

FIG. 4. The THz output spectrum. The solid curve is a calculated spectrum which includes roll off by the carrier lifetime, strip-line losses, the substrate absorption, and the impedance mismatch between the strip line and the dipole antenna.

conductor (Ohmic) loss and radiation loss in the transmission line, and the substrate absorption. The conductor loss ${ }^{9}$ calculated from the skin-effect resistance is $10 \sqrt{f} \mathrm{~dB} / \mathrm{mm}$, where $f$ is the frequency in THz. The radiation loss ${ }^{10}$ is calculated to be $0.6 f^{3} \mathrm{~dB} / \mathrm{mm}$. The substrate absorption coefficient measured with the Fourier transform infrared spectrometer was $\alpha \leqslant 0.5 \mathrm{~mm}^{-1}$ at $\leqslant 3 \mathrm{THz}$. The solid curve in Fig. 4 is the calculated spectrum taking into account the carrier lifetime, the losses described above, and the impedance mismatch between the strip line and the dipole antenna. The mismatch loss was obtained from the strip-line impedance of $76 \Omega$ and the dipole impedance in Ref. 11. The calculation shows rough agreement with the data, but the measured bandwidth is much wider than the calculation, due to the presence of a flat region at 1-2 THz. In order to clarify the details of the device property, further measurements for the devices with various electrode structures and detailed analysis are required.

The bolometer signal of $1 V_{p-p}$ in Fig. 4 corresponds to $\sim 0.1 \mu \mathrm{W}$, while the output power is predicted to be $>10$ $\mu \mathrm{W}$ at $1 \mathrm{THz}$ for a dc photocurrent of $3 \mathrm{~mA}$. This large discrepancy might be due to coupling losses to the bolometer, a shallow modulation depth of the optical interference fringe caused by imperfections in the spatial modes of the lasers, or the space-charge-limited current which does not contribute $\mathrm{THz}$ ac current, but dc current.

In conclusion, a free-space phase-matching technique for traveling-wave photomixers has been proposed and experimentally demonstrated. The device could handle the input laser power of $300 \mathrm{~mW}$, and the device bandwidth was 1.8 THz. The results show that the traveling-wave photomixer has the potential to provide high-power $\mathrm{THz}$ output at frequencies above $1 \mathrm{THz}$ because of its power-handling capability and capacitance-free bandwidth.

The authors would like to thank T. E. Turner in the Microelectronics Device Laboratory at JPL for device fabrication. This research was sponsored by the Jet Propulsion Laboratory, California Institute of Technology, and the National Aeronautics and Space Administration. The work performed at UCSB was supported by the Center for Nonstoichiometric III-V Semiconductors.

${ }^{1}$ E. R. Brown, F. W. Smith, and K. A. McIntosh, J. Appl. Phys. 73, 1480 (1993).

${ }^{2}$ E. R. Brown, K. A. McIntosh, F. W. Smith, K. B. Nichols, M. J. Manfra, C. L. Dennis, and J. P. Mattia, Appl. Phys. Lett. 64, 3311 (1994).

${ }^{3}$ K. A. McIntosh, E. R. Brown, K. B. Nichols, O. B. McMahon, W. F. DiNatale, and T. M. Lyszczarz, Appl. Phys. Lett. 67, 3844 (1995).

${ }^{4}$ S. Matsuura, M. Tani, and K. Sakai, Appl. Phys. Lett. 70, 559 (1997).

${ }^{5}$ S. Verghese, K. A. McIntosh, and E. R. Brown, IEEE Trans. Microwave Theory Tech. 45, 1301 (1997).

${ }^{6}$ L. Y. Lin, M. C. Wu, T. Itoh, T. A. Vang, R. E. Muller, D. L. Sivco, and A. Y. Cho, IEEE Photonics Technol. Lett. 8, 1376 (1996).

${ }^{7}$ Y.-J. Chiu, S. B. Fleischer, and J. E. Bowers, IEEE Photonics Technol. Lett. 10, 1021 (1998).

${ }^{8}$ S. Matsuura, P. Chen, G. A. Blake, J. C. Pearson, and H. M. Pickett, Int. J. Infrared Millim. Waves 19, 849 (1998).

${ }^{9}$ K. C. Gupta, R. Garg, and R. Chadha, Computer-Aided Design of Microwave Circuits (Artech House, Norwood, MA, 1981), p. 72.

${ }^{10}$ D. B. Rutledge, D. P. Neikirk, and D. P. Kasilingam, in Infrared and Millimeter Waves, edited by K. J. Button (Academic, New York, 1983), Vol. 10, p. 1.

${ }^{11}$ M. Kominami, D. M. Pozar, and D. H. Schaubert, IEEE Trans. Antennas Propag. 33, 600 (1985). 